\title{
Empoderamiento de la mujer campesina en el Sur del Tolima1
}

\section{Empowerment of peasant women in southern Tolima}

\section{María del Pilar Salamanca Santos²}

O

Recepción: 14/01/2021
Aprobación: 07/12/2021

Publicación: 22/12/2021

\section{Para citar este artículo}

Salamanca Santos, M. P. (2021). Empoderamiento de la mujer campesina en el Sur del Tolima. Indagare, (9), 74-80.

https://doi.org/10.35707/indagare/907

1 Derivado de los proyectos: Observatorio regional para la construcción de paz y Escuela, Territorio y postconflicto. 2 Psicóloga. Investigadora grupo Zoon Politikon, Facultad de Derecho y Ciencias Políticas de la Universidad de Ibagué, Ibagué, Colombia. ORCID: 0000-0001-7403-1705. Correo electrónico: pilar.salamanca@unibague.edu.co 


\section{Resumen}

Las mujeres rurales son agentes clave para conseguir los cambios sociales, culturales, ambientales y económicos necesarios para el desarrollo de un territorio; enfrentando retos de acceso limitado a créditos, salud y educación. Esto se ve agravado además por las crisis actuales a nivel sanitario y el cambio climático. Su empoderamiento no solo es fundamental para el bienestar de las personas, familias y comunidades rurales, sino también para la productividad económica de la región.

\section{Abstract}

Rural women are key agents in achieving the social, cultural, environmental and economic changes necessary for the development of a territory; facing challenges of limited access to credit, health and education. This is further aggravated by the current health crises and climate change. Their empowerment is not only essential for the well-being of individuals, families and rural communities, but also for the economic productivity of the region.

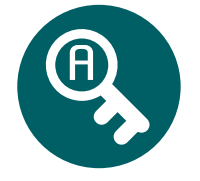

Palabras claves: Mujeres, empoderamiento, territorio.

Key words: Women, empowerment, territory.

\section{Introducción}

La mujer rural ha empezado a ser reconocida como motor de transformación en sus territorios; ellas han asumido una carga excesiva de actividades de cuidado sin remuneración ni reconocimiento, generalmente expuesta a padecer mayores desigualdades y violencias. A causa del conflicto interno del país les tocó aprender a ser fuertes y emprendedoras y, en algunos casos, asumir la jefatura de los hogares, crianza de los hijos y manutención. En medio de este complejo de violencias estructurales, las mujeres han empezado a desarrollar estrategias de supervivencia, de resistencias, pero también de resignificación de su rol en los territorios, acciones que permiten identificarse como emprendimientos locales que reconstruyen tejidos y llegan a convertirse en procesos de construcción de paz (Salamanca \& Uribe, 2019; Cediel, et al., 2017).

En este contexto, marcado por las secuelas del conflicto armado del país, y las desigualdades a las que se ven avocadas las zonas rurales, surgen grupos de mujeres campesinas. Como se ha mencionado, las mujeres han sido actores clave en la recons- 
trucción temprana de sus comunidades, y en la reconstrucción del tejido social, de este modo, es posible evidenciar cómo se han desarrollado importantes iniciativas organizacionales desde la cotidianidad del conflicto y el posconflicto, generando nuevos imaginarios locales que apuestan por la construcción de paz y un repertorio de iniciativas comunitarias (Grupo de Memoria Histórica, GMH, 2009).

\section{Contexto: pasado y presente de la mujer rural}

Lo rural, en los últimos años ha ocupado un lugar central en la agenda pública y en especial en las negociaciones realizadas en La Habana por parte del Gobierno y las FARC, y movimientos de protesta campesina que tuvieron lugar en 2013. En estas negociaciones se reconoce la necesidad de una transformación productiva del campo y las condiciones de vida de los campesinos, siendo el primer punto de acuerdo en los diálogos de paz entre el Gobierno y las guerrillas de las Fuerzas Armadas Revolucionarias de Colombia (FARC). Este primer acuerdo expresa la urgencia de mejorar el acceso y distribución de la tierra, realizar inversiones en vías terciarias, electricidad, comunicaciones e infraestructura de riego y drenaje; aumentar la cobertura y calidad de los servicios de salud, educación, vivienda, agua y saneamiento en el campo, aspectos que aportan a mejorar la calidad de vida en las zonas rurales. Estas barreras que se menciona en el Acuerdo, y que cubren a toda la población campesina, refieren también algunos aspectos específicos que caracterizan y afectan a las mujeres en zonas rurales (Ramírez, et al., 2015). Uno de los factores, que la mujer rural presenta:

Tasas de ocupación muy por debajo de las de los hombres rurales y ganan cerca de la mitad de sus ingresos, estas tienen menos colaterales productivos y monetarios que los hombres. La evidencia sugiere de hecho que existe una brecha de género en los derechos de propiedad (tierras, activos, ahorros) y que la falta de acceso a éstos afecta la equidad en la distribución de la riqueza y acceso a fuentes de financiamiento. (Deere, Oduro, Swaminathan, \& Doss, 2012, citados por Ramírez; et al., 2015, p.10).

De acuerdo al informe Mujeres rurales en Colombia (DANE, 2020), la participación de las mujeres que habitan zonas rurales en el mercado laboral es menor en comparación con los hombres rurales (la tasa de ocupación es menor en 37,3 puntos porcentuales) y con respecto a las mujeres urbanas (la tasa de ocupación es menor en 14,1 puntos porcentuales). Esto puede estar relacionado con una mayor participación de estas mujeres en el trabajo no remunerado. El tiempo diario dedicado a actividades de trabajo no remunerado por parte de las mujeres rurales es mayor que el dedicado por los hombres rurales, con una diferencia de 4 horas con 46 minutos; y mayor que el dedicado a este trabajo por las mujeres urbanas (48 minutos adicionales). 
En este sentido, se ha encontrado que:

Las mujeres rurales presentan más bajos niveles de empoderamiento, mayores niveles de aislamiento y un menor acceso a información que los hombres en zonas rurales. Debido a aspectos culturales patriarcales y machistas, a la falta de oportunidades laborales, la vida diaria de las mujeres en zonas rurales se limita a las labores del hogar, el cuidado de los hijos y el mantenimiento de la unidad productiva que no es remunerado ni reconocido como trabajo. (Martínez-Restrepo, Mejía, \& Enríquez, 2015, citados en Ramírez; et al., 2015, p. 10).

De otra parte, y según la Defensoría Delegada para la Prevención de Riesgos de Violaciones a los Derechos Humanos y el DIH-Sistema de Alertas Tempranas, SAT (2014), en su texto El conflicto armado y el riesgo para la mujer rural:

Las mujeres rurales en Colombia son cuatro veces victimizadas y discriminadas, primero, como resultado de la exclusión e invisibilización de las necesidades que padece el sector rural del que hacen parte, esto como resultado de la desigualdad estructural respecto al mundo urbano (prestación de servicios públicos de calidad, cobertura en educación y salud, etc.); segundo, por su condición de mujer "ya que el tratamiento histórico, cultural y Socioeconómico que recibe, determinado por la estructura patriarcal, es más injusto frente al hombre”. (Defensoría del Pueblo, 2014, p. 14).

De acuerdo con el informe Mujeres rurales: gestoras de esperanza, la violencia se presenta tanto en sus vidas cotidianas, como en el contexto del conflicto armado, producto de las relaciones de género existentes en dicho contexto. Además, resalta que la competencia entre grupos armados por el territorio "ha afectado las formas de socialización campesina y desestabilizado su relación con la tierra” (PNUD, 2011, p. 53).

La violencia contra la mujer, en los últimos 30 años, ha comenzado a ser visibilizada en la agenda pública, lográndose el compromiso de instituciones de carácter local y nacional para la inclusión e implementación de políticas públicas de prevención y protección contra todos los tipos de violencia que afectan a las mujeres. A ello se suma el surgimiento de organizaciones sociales que impulsan la prevención de cualquier tipo de violencia o exclusión desde una perspectiva de género desde los años 80; así como la protección diferencial de las víctimas. Se ha avanzado en la aprobación de normativas y la puesta en marcha de acciones para prevenir las formas de agresión contra las mujeres a manos de personas de su entorno social, familiar o integrantes de estructuras armadas en los distintos espacios públicos y privados. (Defensoría del Pueblo, 2014, p. $15)$. 


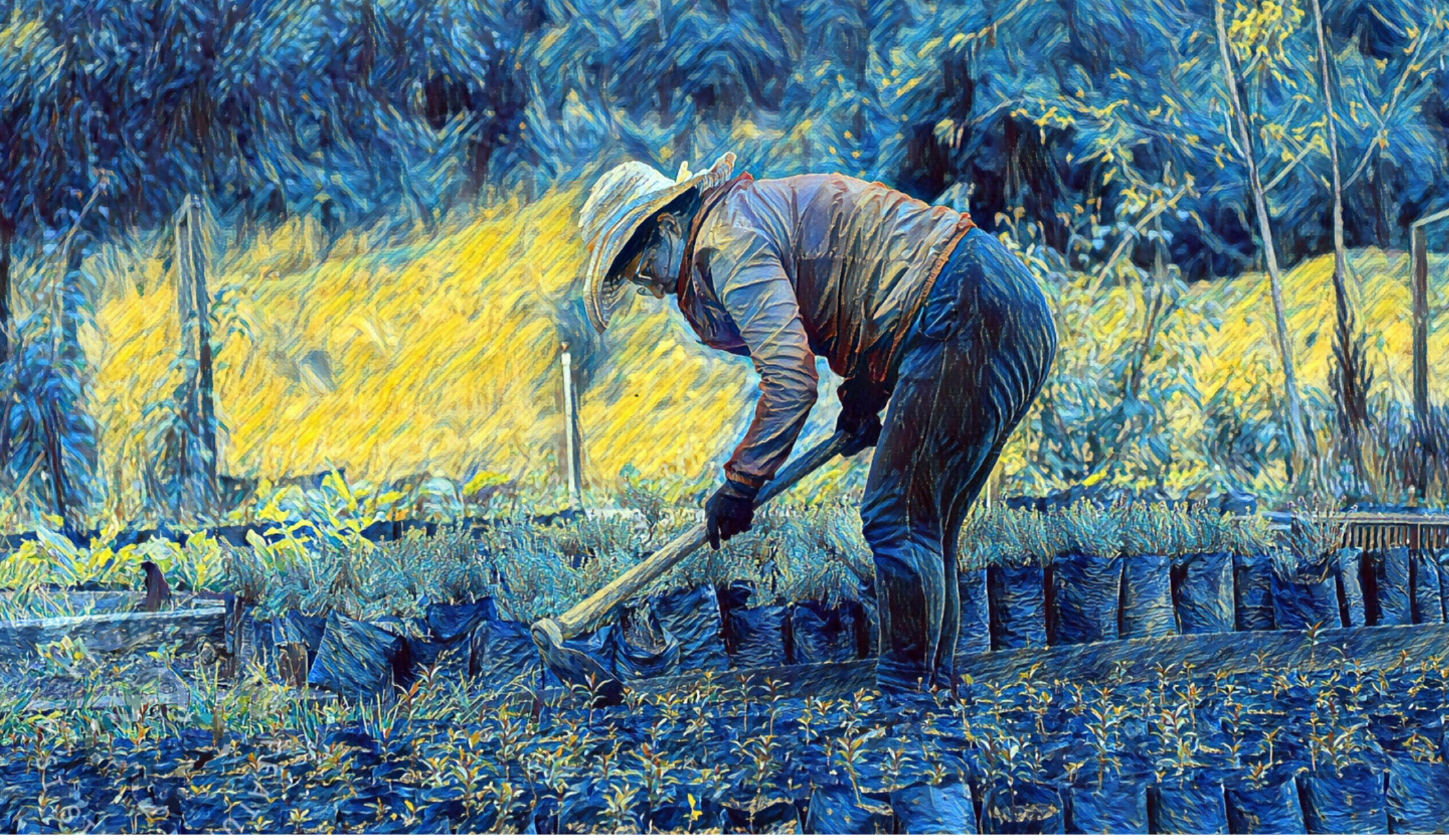

\section{Empoderamientos en el territorio}

El informe del PNUD (2011) reconoce el rol que estos colectivos de mujeres rurales han jugado en las luchas sociales del sector rural y la reivindicación de los derechos del campesinado colombiano, "a pesar de las diversas formas de victimización padecidas, han asumido un papel activo en la reivindicación de derechos” (PNUD, 2011, p. 82).

A pesar de una realidad caracterizada por la exclusión, discriminación y violencia, las mujeres son actoras destacadas de las luchas sociales y políticas en el sector rural y agentes de su propia transformación. Se resalta su gran capacidad de agenciar su vida y las de sus organizaciones, pues se han formado como líderes y participan en diferentes espacios sociales. (PNUD, 2011, p. 20).

Son amplios los ejemplos de organización de las mujeres del Sur del Tolima, en espacios de incidencia social y áreas productivas; es así que en el periódico de circulación nacional El Tiempo, en su artículo Mujeres aportan un grano de café a la paz, se resalta el trabajo de 300 mujeres que hacen parte de las siete asociaciones de mujeres cafeteras suscritas al proyecto "Mujeres productoras de café construyen paz en tres regiones afectadas de Colombia". Se destaca que el café que cultivan estas mujeres es reconocido entre otros por ser "suave, de taza limpia, acidez relativamente alta, aromático y un perfil sensorial de alta calidad" (Equipo Limpal Colombia, 2017, s.p.). Este trabajo realizado por mujeres emprendedoras contribuye además a la construcción de paz desde los territorios, con perspectiva de género. 
En esta línea, en el Sur del Tolima cada vez más aparecen asociaciones cafeteras lideradas y compuestas por mujeres, que evidencian la resiliencia y empoderamiento de las mujeres a pesar de las adversidades propias de las zonas rurales, sumadas al conflicto armado en el territorio. Se pueden referenciar experiencias como la de Asociación de Productoras de la vereda Organizada de Calarma, APROVOCAL, en Risalda, Chaparral, grupo que cuenta con logros de tipo organizacional, comercial y social, que les ha valido el reconocimiento público otorgado por entidades locales, departamentales y nacionales.

APROVOCAL en su actividad productora de Café de origen Calarma, va más allá, ya que comparten su conocimiento de producción y proceso con el colegio de su localidad, en la que los estudiantes hacen una práctica de laboratorio; sumado a esto, vienen trabajando en una actividad de ecología responsable mediante la reforestación de una de las cuencas de la zona, para ello han generado un vivero con plantas nativas, a las que la comunidad tiene acceso. Cabe resaltar otras organizaciones de mujeres en el municipio de Chaparral, como: ASMUES, ASOMUCA, ASMUR, ASOMUPRO, ASMUIRCO, entre otras.

En torno a la producción y comercialización del café se forjan costumbres, creencias, gustos por los sabores y aromas y toda una tradición, que con el tiempo y lucha ha permitido visibilizar el trabajo de la mujer cafetera y su papel en el desarrollo de las regiones (Equipo Limpal Colombia, 2017). Mujeres que a través de la cadena productiva del café se empoderan, aprenden a defender y a ejercer sus derechos como mujeres y como caficultoras.

\section{Conclusión}

La resistencia de la mujer campesina del Sur del Tolima, en tiempos de postconflicto, generará nuevas oportunidades y emprendimientos para otras mujeres que buscan mejorar su calidad de vida y la de sus familias, por medio de proyectos e iniciativas de negocio; es así que se reconoce el poder que poseen las mujeres para transformar sus comunidades y su lucha por los espacios que visibilicen su rol en la sociedad.

Las actividades desarrolladas por las organizaciones de mujeres rurales son de gran importancia para el desarrollo de los territorios, en las que su labor aporta a la disminución de la pobreza; motivación para otras mujeres en la planeación de proyectos de vida individuales y colectivas; con posibilidad de generar espacios para las nuevas generaciones, donde el campo tiene futuro. Sin embargo, no se puede negar que, a pesar de los avances y logros de las mujeres rurales, siguen siendo víctimas de la inequidad de género, de la discriminación y de la violencia cultural y estructural presentes en los territorios. 


\section{Referencias}

Cediel Becerra, N. M., Hernández Manzanera, J., López Duarte, M. C., Herrera Buitrago, P., Donoso Burbano, N., \& Moreno González, C. (2017). Empoderamiento de las mujeres rurales como gestoras de los Objetivos de Desarrollo Sostenible en el posconflicto colombiano. Equidad y Desarrollo, (28), 61-84. https://doi.org/10.19052/ed.4077

Defensoría del Pueblo. (2014). El conflicto armado y el riesgo para la mujer rural. Estudios de caso en los departamentos de Chocó, Córdoba, Santander y Caquetá. Recuperado de https://colombia.unwomen.org/es/biblioteca/publicaciones/2014/09/libro-defensoria

Departamento Administrativo Nacional de Estadística, DANE. (2020). Mujeres rurales en Colombia. Recuperado en https://www.dane.gov.co/files/investigaciones/notas-estadisticas/sep-2020-\%20mujeres-rurales.pdf

Equipo Limpal Colombia. (2017). Mujeres aportan un grano de café a la paz. El Tiempo. Recuperado de https://www.eltiempo.com/colombia/otras-ciudades/mujeres-aportan-un-grano-de-cafe-a-lapaz-106050

Grupo de Memoria Histórica, GMH. (2009). Memorias en tiempos de guerra. Repertorio de iniciativas. Bogotá. Colombia: Puntoaparte Editores.

Programa de las Naciones Unidas para el Desarrollo, PNUD. (2011). Mujeres rurales: gestoras de esperanzas. Recuperado de https://europa.eu/capacity4dev/public-gender/document/mujeres-rurales-gestoras-de-esperanza

Ramírez, J.M., Martínez-Restrepo, S., Sabogal, A., Enríquez, E., Salas, R., \& Rodríguez, V. (2015). Barreras de acceso de la mujer rural a crédito, programas asociativos y a la formalización de la tierra en el norte del cauca y el sur del Tolima. Recuperado de https://www.repository.fedesarrollo.org.co/ handle/11445/2725

Salamanca Santos, M. P., \& Uribe Sarmiento, J. J. (2019). Aprendiendo a ser fuertes y emprendedoras. Estrategias de supervivencia en mujeres desplazadas víctimas del conflicto armado. En Gobierno y gobernanza. Desplazamiento forzado, análisis para la prevención y la restauración de los vínculos sociales rotos (pp. 49-77). Bogotá, Colombia: Universidad Externado de Colombia. 